\title{
СТРУКТУРНЫЕ АСПЕКТЫ ЦИКЛИЧЕСКОЙ ПРОЧНОСТИ СВАРНОГО УЗЛА ПАРОПЕРЕГРЕВАТЕЛЯ
}

\author{
Заворин Александр Сергеевич ${ }^{1}$, \\ zavorin@tpu.ru
}

\section{Любимова Людмила Леонидовна', II@tpu.ru}

\author{
Ташлыков Александр Анатольевич 1 , \\ tashlykov@tpu.ru
}

\author{
Буваков Константин Владимирович', \\ kvb@tpu.ru \\ 1 Национальный исследовательский Томский политехнический университет,
Россия, 634050, г. Томск, пр. Ленина, 30.
}

\begin{abstract}
Актуальность исследования сварных узлов теплогенераторов связана с необходимостью увеличения надежности работы часто повреждаемых и наиболее ответственных узлов конструкций теплоэнергетического оборудования. Одним из факторов увеличения ресурса работоспособности является учет внутренних структурных напряжений, механизм формирования которых связан с развивающейся в процессе эксплуатации структурной неоднородностью и ресурсной структурной деградацией, приводящей к разрушению.

Цель исследования: установление связей между накоплением структурной поврежденности, внутренними напряжениями и трещинообразованием под влиянием механических циклических нагрузок.

Объект: сварной узел пароперегревателя, выполненный из низколегированной жаропрочной стали 12Х1МФ.

Методы: физическое моделирование условий эксплуатации путем внешнего механического циклического деформирования, микроскопическое исследование морфологии поверхности, рентгенометрия деформированных образцов, оценка внутренних структурных напряжений, среднеквадратичных смещений атомов при деформировании и характеристической температуры. Результаты. Показана роль и влияние циклической нагрузки на разрушение, заключающиеся в волновом периодическом деформационном «упрочнении-разупрочнении» и в циклическом изменении внутренних структурных напряжений. Установлено, что в условиях переменного циклического нагружения характерным процессом является периодическая релаксация внутренних напряжений, соответствующая разрыву межатомных связей и образованию микроструктурно короткой трещины. По условиям релаксации, в соответствии с представлениями А. Гриффитса, установлено критическое напряжение раскрытия трещины. Значения среднеквадратичных смещений атомов и характеристическая температура при циклическом деформировании отражают структурные признаки пластичности, пористости и хрупкого состояния.

Выводы. Предложена новая методология оценки предельных состояний околошовной зоны сварных соединений на основе использования установленных связей между внутренними напряжениями и накоплением структурной повреждаемости. Показано, что внутренние напряжения, колебательная амплитуда атомов $\widetilde{U}^{2}$ и характеристическая температура могут служить диагностическим признаком структурных превращений: признаком хрупкого разрушения является глубокая (до нуля) релаксация внутренних напряжений I рода; структурный признак пластичности проявляется повышенной амплитудой атомных смещений $\bar{U}^{2} ;$ a структурным признаком пористости является аномальное повышение колебательной амплитуды атомов $\bar{U}^{2}$ и уменьшение характеристической температуры.
\end{abstract}

\section{Ключевые слова:}

Сварной узел, сталь, пароперегреватель, внутренние напряжения, механические циклические нагрузки, структурная деградация, трещинообразование, циклическая прочность, среднеквадратичные смещения атомов, характеристическая температура.

\section{Введение}

Число аварийных ситуаций от усталости околошовных зон (ОШЗ) и зон термического влияния сварки (ЗТВ) остается весьма значительным и увеличивается пропорционально росту применения сварных конструкций [1-7].

Прочность и долговечность сварных соединений достигается рациональным выбором конструктивных, металлургических и технологических средств. Тем не менее, требуемая работоспособность и должные эксплуатационные качества сварного соединения обеспечиваются далеко не всегда.

В конструкциях сварных узлов расчет прочности производят по допускаемым напряжениям. Их определяют как частное от деления напряжения, условно принимаемого за разрушающее, на коэффициент запаса прочности.

Пониженные свойства сварных соединений при расчетах на прочность учитываются через коэффициенты запаса прочности, значения которых находятся в диапазоне $0,4-1,0$ и определяются следующим образом $[2,8,9]$.

Разупрочнение металла шва оценивается коэффициентом

$$
\varphi_{\omega}^{\mathrm{M} \amalg}=\frac{\left(\sigma_{\omega}^{\mathrm{M}}\right)_{\mathrm{C}}}{\sigma_{\text {дा }}^{\mathrm{OM}}},
$$

где $\left(\sigma_{\omega}^{\text {мш }}\right)_{\mathrm{C}}$ - длительная прочность металла шва в сварном соединении с учетом контактного упроч- 
нения; $\sigma_{\text {дп }}^{\text {м }}$ длительная прочность основного металла.

При этом

$$
\left(\sigma_{\omega}^{\mathrm{M} \amalg}\right)_{\mathrm{C}}=\sigma_{\omega}^{\mathrm{M} \amalg} \mathbf{K}_{\chi} \mathbf{K}_{\mathrm{P}},
$$

где $\sigma_{\omega}^{\text {мш }}$ - длительная прочность металла шва в сварном соединении без учета контактного упрочнения; $\mathrm{K}_{\chi}=\frac{\pi}{4}+\frac{1+2 \gamma}{3 \sqrt{3(1+\gamma)}} ; \gamma$ - геометрическая характеристика, равная $\gamma=\frac{D_{\mathrm{H}}+D_{\mathrm{BH}}}{2 D_{\mathrm{H}}}$, где $D_{\mathrm{H}}$, $D_{\text {ВП }}$ - наружный и внутренний диаметр стенки трубы ответственно; $\mathrm{K}_{\mathrm{p}}-$ коэффициент, который в области значений $\chi=\chi_{\text {дп }}$ равен $\mathrm{K}_{\mathrm{p}}=1,0$, а в области

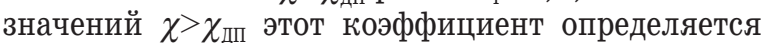
как $\mathrm{K}_{\mathrm{P}}^{\mathrm{MIN}}=1,25-0,25 \mathrm{~K}_{\text {дп }}$. В этом выражении

$$
\mathbf{K}_{\text {дा }}=\frac{\sigma_{\text {дП }}^{\mathrm{OM}}}{\sigma_{\omega}^{\mathrm{M \amalg}}},
$$

где $\sigma_{\text {дा }}^{\mathrm{Mш}}, \sigma_{\omega}^{\mathrm{Mш}}-$ длительная прочность основного металла и металла шва; $\chi$ - относительная ширина мягкой прослойки металла шва, равная $\chi=F / S^{2}$ $(F-$ площадь металла шва в поперечном сечении, $S$ - толщина стенки в зоне сварного шва). $\chi_{\text {дп }}-$ критическая относительная толщина мягкой прослойки металла шва, при которой ее длительная прочность в сварном соединении составляет:

$$
\chi_{\text {дп }}=\left(\frac{4 \mathrm{~K}_{\text {дП }}}{\mathrm{K}_{\mathrm{P}}}-\pi\right)^{-1} .
$$

Из изложенного следует, что все оценки сопротивления разрушению металла сварного шва основываются на однородности, сплошности, изотропности материла, индивидуальные свойства которого не учитываются, принимаются одинаковыми во всех его точках и по всем направлениям; материал как бы априори является бесструктурным и не имеет дефектов.

Не учитываются также изменения, происходящие в теле при превышении в них предела упругости, не учитывается влияние микроструктуры и других характеристик, связанных с атомно-дислокационной теорией строения твердого тела, не отражаются превращения при термомеханических внешних возмущениях [10].

Все это не позволяет предвидеть направление изменения расчетных значений допускаемых напряжений, которые по порядку величины могут не соответствовать реально действующим в конструкции. Очевидно, что будут иметь место серьезные противоречия между выводами, вытекающими их представлений о макроскопическом континууме, и представлениями, основанными на атомистическом подходе к таким явлениям, как разрушение.

Этот недостаток может быть устранен путем учета внутренних структурных напряжений, механизм формирования которых связан с перечисленными факторами структурной неоднородности и ресурсной структурной деградации.
Внутренние напряжения в большинстве случаев играют отрицательную роль, так как далеко не всегда удаётся получить их благоприятное распределение по объёму изделия [11-13].

Однако все-таки очевидно, что учет внутренних напряжений является резервом прочности в машиностроении и основой прогнозирования ресурса [14].

Цель исследования направлена на установление связей между накоплением структурной поврежденности, внутренними напряжениями и трещинообразованием под влиянием механических циклических нагрузок.

Образеи для исследований вырезался из участка трубы в районе обмуровки котла в точке Б (рис. 1). Трубы изготовлены из стали $12 \mathrm{X} 1 \mathrm{M \Phi} \mathrm{в}$ 1999 г. Сварной узел выполнен в 2005 г. Трубы эксплуатировались в котле 16500 ч. Рабочая температура в точке Б составляла $480{ }^{\circ} \mathrm{C}$. Труба была снята с эксплуатации из-за разрушения в точке $\mathrm{A}$, где поток дымовых газов обладал высокой скоростью и температура достигала порядка 540-590 ․

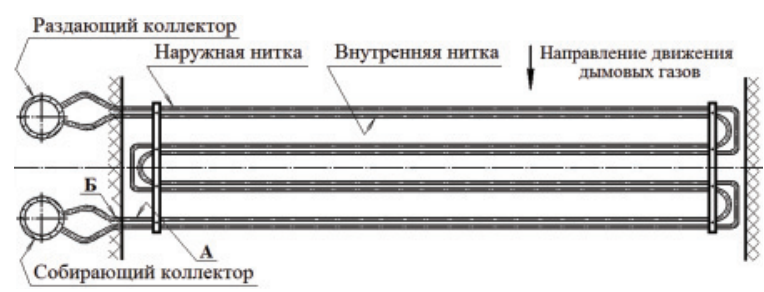

Рис. 1. Вид сверху на горизонтальный пароперегреватель парового котла

Fig. 1. Top view of the horizontal steam boiler superheater

Исследованию подвергалась околошовная зона однородного сварного узла, выполненного из низколегированной жаропрочной стали $12 \mathrm{X} 1 \mathrm{MФ.} \mathrm{0б-}$ разец для исследований вырезался на расстоянии 7,5 мм от оси сварного шва (рис. 2).

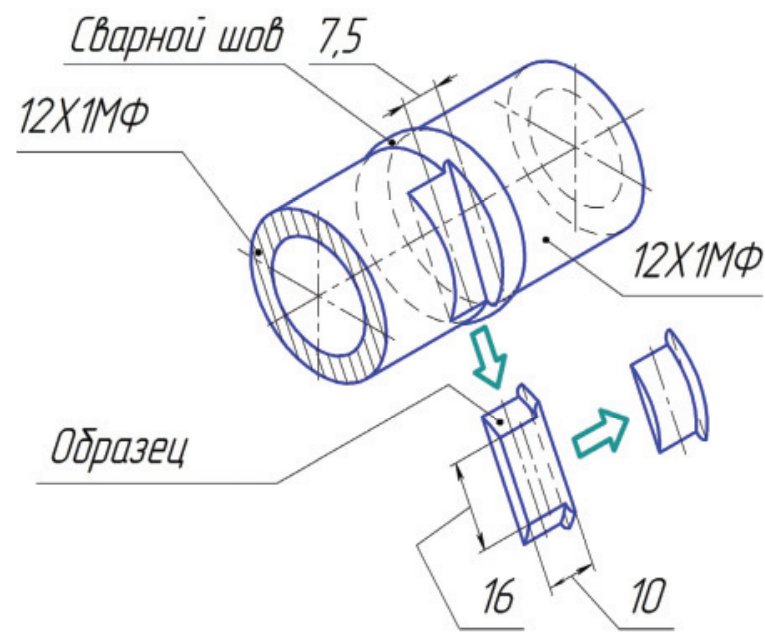

Рис. 2. Схема вырезки образца из околошовной зоны однородного сварного узла из стали $12 Х 1 M Ф$

Fig. 2. Scheme for cutting a sample from the weld-affected zone of a homogeneous weldment made of $12 \mathrm{Cr} 1 \mathrm{MoV}$ steel 
В качестве образца-свидетеля (эталона) применялся специально подготовленный идеально-мозаичный поликристаллический образец из стали 12Х1МФ.

Кроме этого, из основного металла прямого участка трубы из стали $12 \mathrm{X} 1 \mathrm{MФ} \mathrm{было} \mathrm{изготовлено}$ четыре контрольных образца 1,1 ', 3 , 3', не подверженных термическому влиянию сварки. Образцы 1' и 3' полировались до зеркального блеска. Образцы 1 и 3 неполированные.

\section{Методика}

Методика испытаний заключалась в организации механического циклического деформирования образца с повышением нагрузки в каждом очередном цикле нагружения. Всего выполнено 13 циклов нагружения с шагом порядка $50 \mathrm{MПа.}$

При этом рентгеновским методом по изменению параметра кристаллической решетки на основании закона Гука производилась оценка макронапряжений I рода (зональных) [15, 16]:

$$
\frac{\Delta l}{l}=\frac{\sigma}{E} \text { или } \frac{\Delta a}{a}=\frac{\sigma}{E},
$$

где $\Delta a / a$ - относительное удлинение параметра кристаллической ячейки при растяжении; $\sigma$ внутренние напряжения I рода; $E$ - модуль нормальной упругости.

Среднеквадратичные смещения атомов $\bar{U}^{2}$ при деформировании определялись по экспериментальным значениям интегральных интенсивностей дифракционных линий образца и эталона на основании выражения [8, 9]:

$$
\bar{U}^{2}=\frac{3}{4 \pi^{2}}\left(\frac{d}{n}\right)^{2} \ln \left(\frac{I}{I^{\prime}}\right),
$$

в котором $n$ - порядок отражения; $d$ - межплоскостное расстояние, А̊; $I^{\prime}$ - интегральная интенсивность для деформированного образца; $I$ - интегральная интенсивность для эталона.

По величине среднеквадратичных смещений $\bar{U}^{2}$ оценивалась характеристическая температура $\Theta$ [15]:

$$
\bar{U}^{2}=\frac{3 h^{2} T}{k m \Theta^{2}} \rightarrow \Theta=\sqrt{\frac{3 h^{2} T}{k m \bar{U}^{2}}},
$$

где $\bar{U}^{2}$ - среднеквадратичное смещение атомов, $\mathrm{m}^{2}$; $h$ - постоянная Планка; $k$ - постоянная Больцмана; $m$ - масса атома железа; $T=295 K\left(t=22{ }^{\circ} \mathrm{C}\right)-$ температура.

Видно (1), что $\bar{U}^{2} \sim 1 /\left(m \Theta^{2}\right)$. В то же время величина $\left(m \Theta^{2}\right)$ характеризует прочность межатомной связи, поскольку она пропорциональна силе $F$, возвращающей атомы в положение равновесия: $F \sim m \Theta^{2}$.

Методика эксперимента включала, кроме измерений внутренних напряжений I рода, оценку ОКР (областей когерентного рассеяния) Д при деформировании.

Идеализированное представление о строении кристалла сводится к тому, что под кристаллитом понимаются некие отдельные, почти идеальной структуры блоки нанометрических размеров, так называемые области когерентного рассеяния (ОКР), из которых состоит зерно. Кристаллиты не развернуты относительно друг друга. Их границы хорошо согласованы (когерентны). Между зернами находятся большеугловые границы, дефектные не только по структуре, но и по составу. В каждом зерне строение блоков (кристаллитов) близко к мозаичному. Таким образом, мозаичный кристалл это агрегат, состоящий из идеальных областей когерентного рассеяния. Интегральная интенсивность дифрагированного пучка от такого кристалла равна сумме интенсивностей, рассеиваемых от каждого блока $I_{\text {моз }}=\Sigma I_{\text {бл. }}$.

В реальном кристалле области когерентного рассеяния по-разному ориентированы по отношению друг к другу, разделены несовершенными по структуре границами, связывающими ОКР, имеют разброс по дисперсности ОКР, по форме частиц, по химическому составу частиц, т. е. по коэффициенту ослабления. При этом условия когерентной дифракции нарушаются, и не только растет ширина дифракционного максимума, но и меняется его интегральная интенсивность. На этом факте основана методика измерения размеров кристалла, когда реальная интегральная интенсивность сравнивается с той, которая могла бы быть у мозаичного кристалла, если его интенсивность была бы уменьшена на некоторый поправочный коэффициент, эквивалентный увеличению коэффициента ослабления. Этот коэффициент называется коэффициентом первичной экстинкции.

В реальных кристаллах интегральная интенсивность дифракционной линии может быть выражена уравнением $[15,16]$ :

$$
I_{\mathrm{P}}=I_{\mathrm{MO3}} \frac{\operatorname{th}(n q)}{n q},
$$

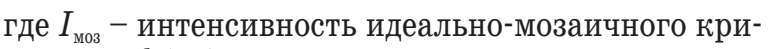
сталла; $\operatorname{th}(n q) / n q$ - коэффициент первичной экстинкции; $\operatorname{th}(n q)$ - гиперболический тангенс; $n$ число параллельных отражающих атомных плоскостей в одном блоке; $q$ - отражательная способность, отнесенная к одной атомной плоскости, определяемая по выражению $[15,16]$ :

$$
q=\frac{1}{2} \frac{e^{2}}{m c^{2}} \frac{\rho}{A} N_{o} \frac{d_{h k l} \lambda}{\sin \theta} F,
$$

где $e^{4} / 2 m^{2} c^{4}=7,83 \cdot 10^{-26}, \mathrm{~cm}^{2} ; \rho$ - плотность вещества; $A$ - атомный вес; $N_{o}$ - число Авогадро; $d_{h k l}-$ межплоскостное расстояние; $\lambda$ - длина волны используемого излучения; $\theta$ - угол дифракции; $F$ структурная амплитуда.

Тогда размер области когерентного рассеяния Д может быть определен из условия $[15,16]$ :

$$
\text { Д }=n d_{h k l} \text {. }
$$

Для реализации методики специально подготавливался идеально-мозаичный эталон, по интегральной интенсивности которого $I_{\text {моз }}$, в соответствии с выражением (2), устанавливалось значе- 
ние $\operatorname{th}(n q) / n q$, по которому определялась величина произведения $(n q)$, а по нему число атомных плоскостей в одном блоке $n$, попадающих в отражающее положение.

Структурная амплитуда $F$ в выражении (3) при известной структуре и координации базисных атомов в элементарной объемноцентрированной кубической ячейке (ОЦК) альфа-фазы стали $12 \mathrm{X} 1 \mathrm{M} \Phi$ определяется следующим образом.

Положение базисных атомов в ОЦК-ячейке определяется как [000], [1/2,1/2,1/2]. Структурная амплитуда F равна:

$$
F=\sum_{i=1}^{i=q} f_{i} e^{2 \pi i\left(H m_{i}+K n_{i}+L p_{i}\right)},
$$

где $H, K, L$ - индексы интерференции линий $(H K L)$ и координаты базисных атомов $m_{i}, n_{i}, p_{i} ; f_{i}-$ амплитуда атомного рассеяния.

Выражение (4) с использованием формулы Эйлера для дифракционного рефлекса (110) можно переписать в следующем виде:

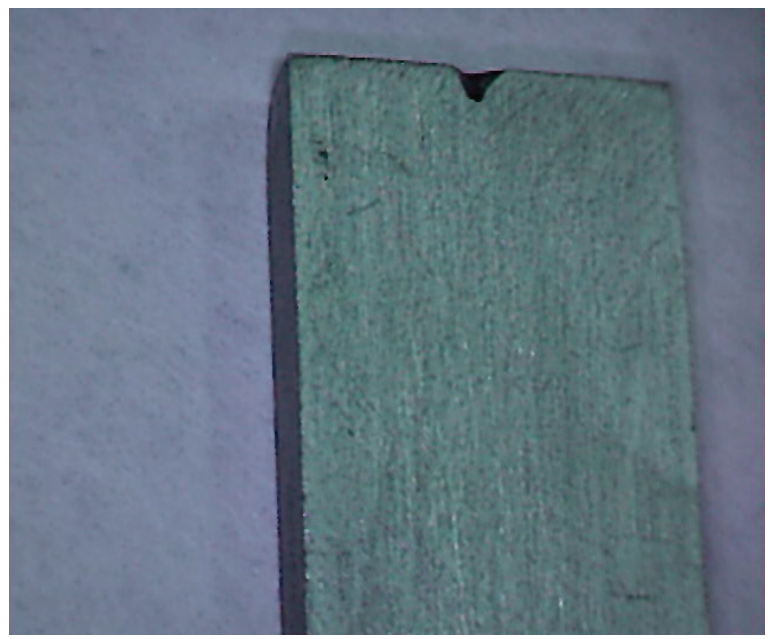

$a$

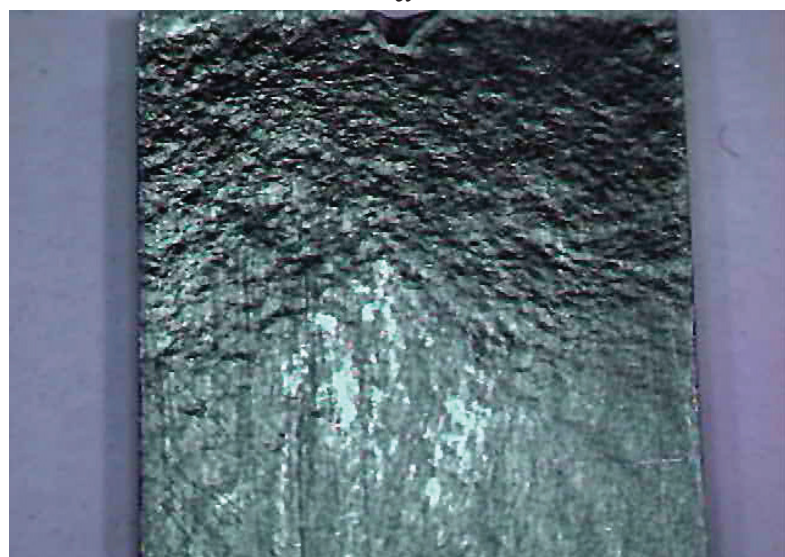

$c$

$$
\begin{gathered}
f_{i} \cdot e^{2 \pi i\left(\mathrm{Hm}_{i}+K n_{i}+L p_{i}\right)}= \\
F_{110}=\sum_{i=1}^{i=q}=f_{\mathrm{Fe}(110)}\left[e^{2 \pi i(1 \cdot 0+1 \cdot 0+0 \cdot 0)}+e^{2 \pi i\left(1 \cdot \frac{1}{2}+1 \cdot \frac{1}{2}+0 \cdot \frac{1}{2}\right)}\right]= \\
=f_{\mathrm{Fe}(110)}\left[\begin{array}{l}
(\cos 2 \pi \cdot 0+i \cdot \sin 2 \pi \cdot 0)+ \\
+(\cos 2 \pi \cdot 1+i \cdot \sin 2 \pi \cdot 1)
\end{array}\right]= \\
=f_{\mathrm{Fe}(110)}[(\cos 0+i \cdot \sin 0+\cos 2 \pi+i \cdot \sin 2 \pi)]= \\
=f_{\mathrm{Fe}(110)}(1+0+1+0)=2 f_{\mathrm{Fe}(110)} .
\end{gathered}
$$

Функция атомного рассеяния вычислялась интерполяцией по справочным табличным данным.

Техника эксперимента. Для экспериментальных исследований использован рентгеновский дифрактометр типа ДРОН с медным антикатодом при селективной фильтрации медного $C u K_{\beta}-$ излучения рентгеновской трубки с применением никелевого селективно-поглощающего $\beta$-фильтра.

Структура поверхности образца наблюдалась с помощью микроскопа PENS COPE при 10-кратном увеличении.

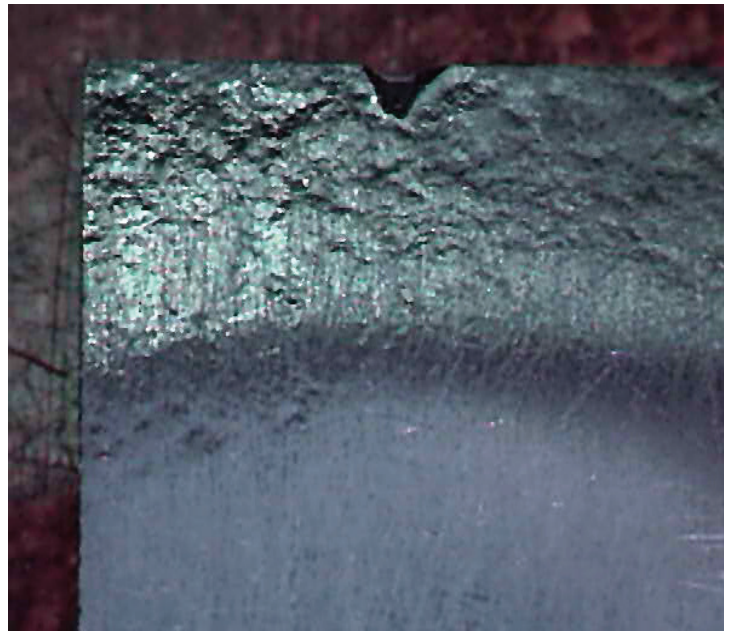

$b$

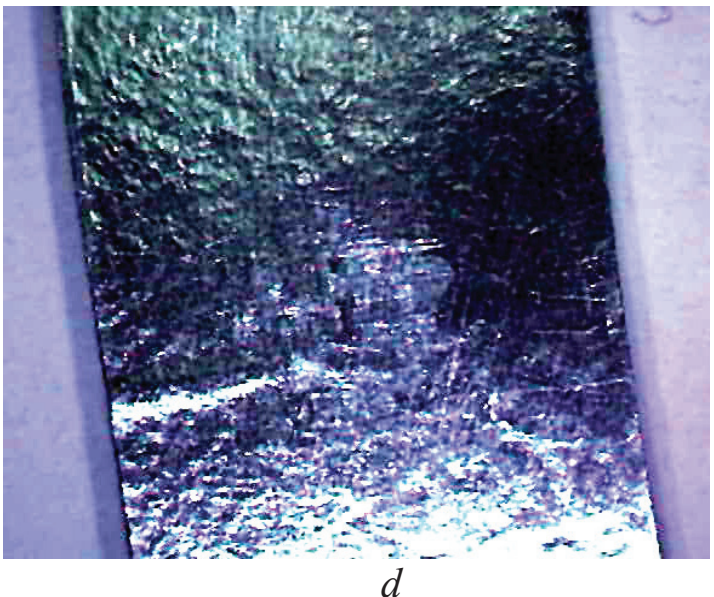

Рис. 3. Тестируемые области деформируемого образца; ×10: а) исходный образец; $b, c, d)$ после деформирования

Fig. 3. Test areas of the deformable specimen; $\times 10: a)$ initial specimen; $b, c, d)$ specimen after deformation 


\section{Экспериментальные результаты}

На рис. 3 представлены некоторые фрагменты структуры поверхности образца и наблюдаемые при деформировании эффекты.

В результате приложения к образцу внешнего возмущающего воздействия на поверхности наблюдается следы необратимой сдвиговой деформации. Микрополоса пластического сдвига распространяется от середины к краям образца, рассеивается на твердых неоднородностях - устойчивых макрообъемах (кластерах), и огибает их. За неоднородностями пластические волны интерферируют, создавая рельеф в виде интерференционных колец (рис. $3, b, c$ ).
Поверхность деформируемого образца приобретает ямочный характер (рис. $3, c$ ). Важнейшей особенностью процесса пластического течения на макроскопическом уровне является его склонность к фрагментации и локализации, которая начинается одновременно с пластическим теченем и заканчивается в момент разрушения образца, что позволяет по особенностям развития локализации пластической деформации устанавливать стадию предразрушения материала, когда образование макроскопических очагов пластической локализации завершается их слиянием в момент разрушения.

Область, расположенная впереди фронта полосы пластического сдвига, претерпевает деформа-

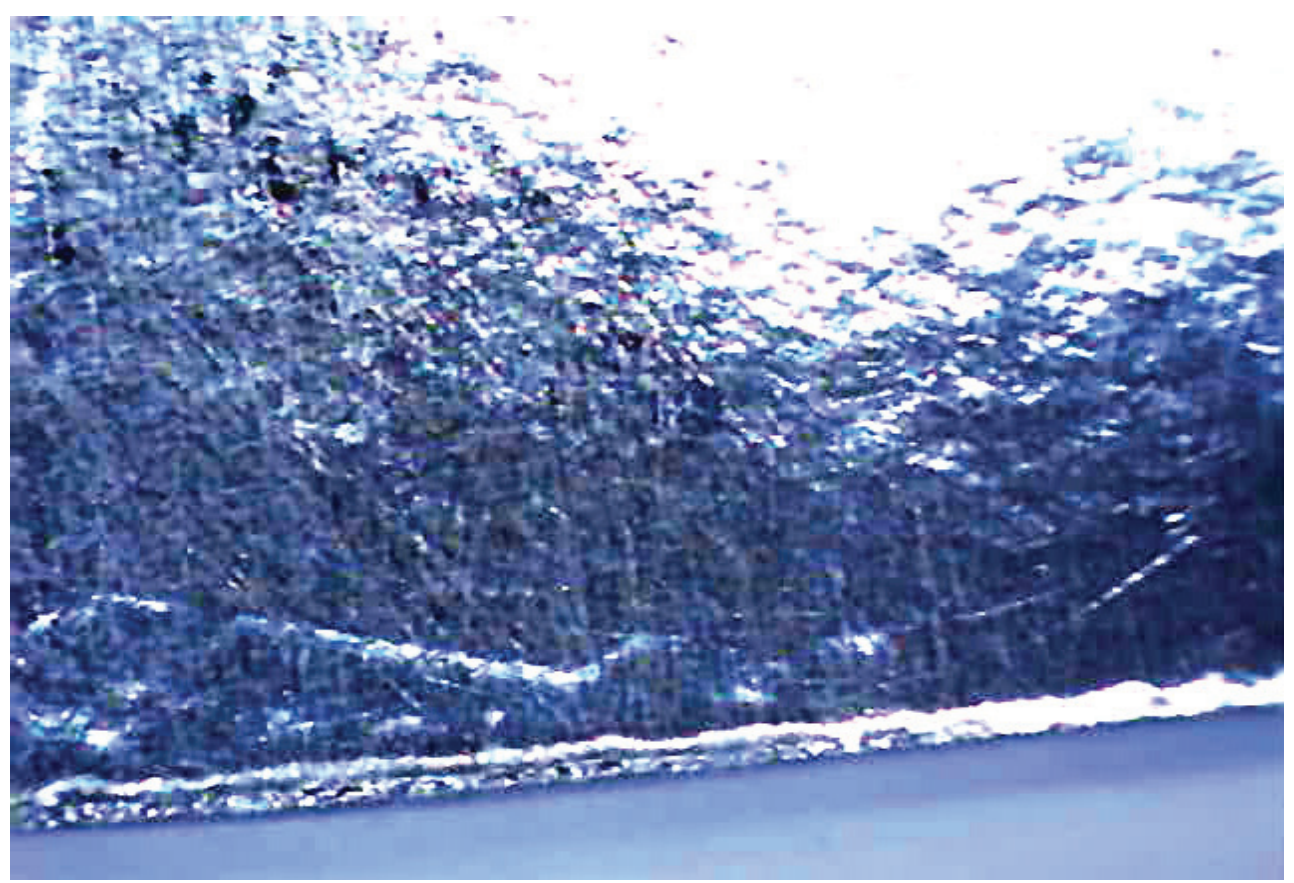

Рис. 4. Развитие пористости в деформируемом образце; $\times 10$

Fig. 4. Porosity development in the deformable specimen; $\times 10$

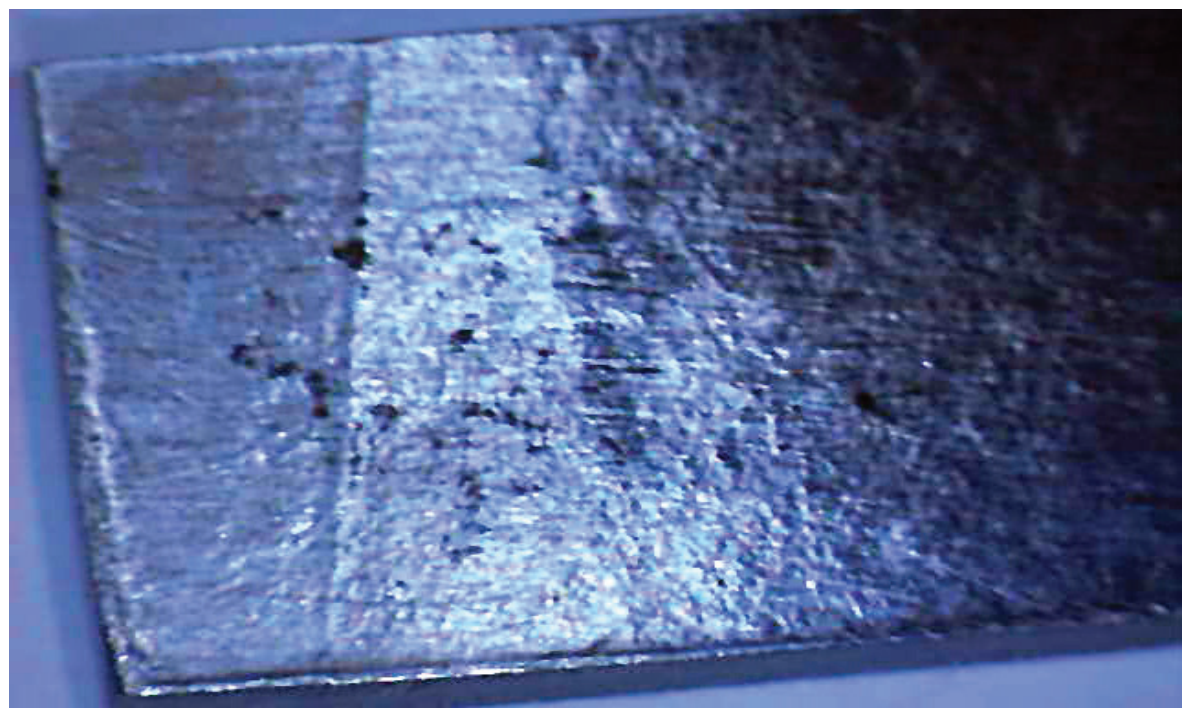

Рис. 5. Распространенная пористость, цепочки пор, сдвиговая волна, продольные хрупкие трещины х10

Fig. 5. Common porosity, pore chains, shear wave, longitudinal brittle cracks; $\times 10$ 
ционное упрочнение (наклеп). В какой-то момент времени микрополоса сдвига не может преодолеть зону упрочнения и тормозится, визуализируясь на поверхности в виде волн, имеющих гребень и впадину, что определяется способностью материала к пластическому течению (рис. $3, d, 4)$.

Дальнейшее приложение внешней нагрузки приводит к возникновению единичных разрушений в виде довольно крупных пор (рис. 4).

В последующих циклах нагружения отдельные поры на поверхности образца сливаются, образуя магистральные трещины (рис. 5).

Движущей силой перемещения полосы скольжения от центра к краям образца могут быть градиенты дефектов и градиенты напряжений. Границы образца обладают повышенной дефектностью и наличием градиента концентрации вакансий. Массоперенос происходит по направлению к граням образца, а диффузия вакансий идет из зон с их повышенной концентрацией в зону, свободную от них. Поэтому в центральных частях образца возникают зоны диффузионной пористости (рис. 5). Наблюдается как бы своеобразная ползучесть как процесс диффузионного течения. Другим фактором перемещения полосы пластического сдвига могут быть неоднородные внутренние напряжения как самостоятельная движущая сила диффузии.

Таким образом, в условиях переменного циклического нагружения наблюдается взаимодействие одновременно протекающих разных по своей физической природе процессов - хрупких разрушений и пластического течения, отражающих два фундаментальных состояния металла (стали).

Роль и влияние циклической нагрузки на разрушение заключается в волновом периодическом деформационном «упрочнении-разупрочнении», что подтверждается результатами циклического изменения внутренних структурных напряжений (рис. 6, 7).

Согласно рис. 6,7 , можно отметить два типа взаимосвязанных процессов, согласованно протекающих в деформируемой среде. С одной стороны, это релаксационные процессы, возникающие за счет движения дислокаций, т. е. пластическое релаксационное формоизменение. С другой стороны, каждый акт пластичности сопровождается генерацией упругих волн, связанной с перераспределением упругих деформаций в ходе релаксационных актов деформации.

Характерным процессом при деформировании является периодическая релаксация внутренних напряжений (рис. 6, 7). Релаксация внутренних напряжений за счет разрыва межатомных связей соответствует процессу разупрочнения и характеризует момент образования микроструктурно короткой трещины [17-19]. Известно, что процесс разрыва межатомных связей является ведущим на всех стадиях разрушения [18-24].

Последствием разрушения межатомных связей является релаксация напряжений и трещинообразование [19, 25].
$\sigma_{\mathrm{I}}, \mathrm{M \Pi а}$

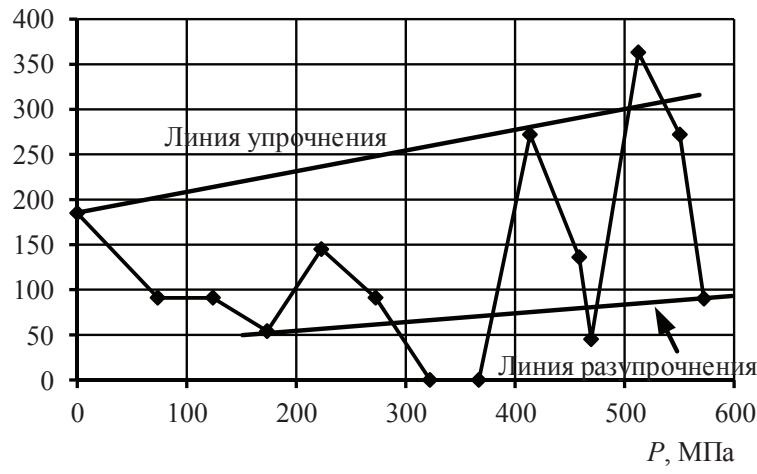

Рис. 6. Распределение внутренних напряжений первого рода $\left(\sigma_{l}\right)$ в околошовной зоне сварного шва (наружная сторона образца)

Fig. 6. Distribution of the first kind internal stresses $\left(\sigma_{l}\right)$ in the weld-affected zone near the weld zone (outer side of the specimen)

$\sigma_{\mathrm{I}}, \mathrm{M \Pi а}$

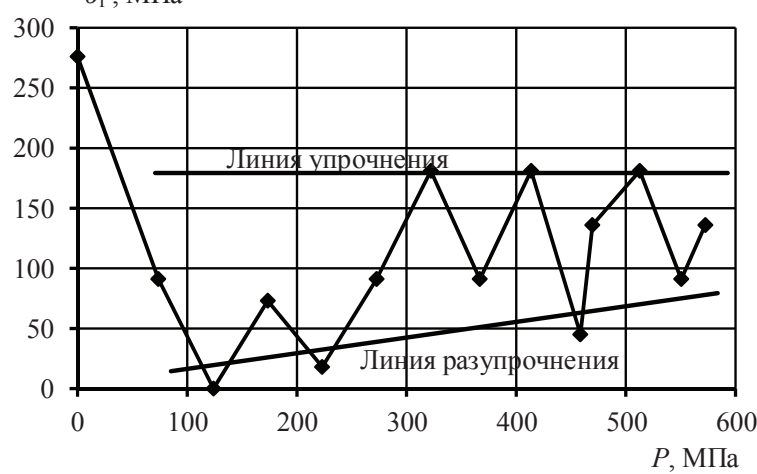

Рис. 7. Распределение внутренних напряжений первого рода $\left(\sigma_{l}\right)$ в околошовной зоне сварного шва (внутренняя сторона образца)

Fig. 7. Distribution of the first kind internal stresses $\left(\sigma_{l}\right)$ in the weld-affected zone near the weld zone (inner side of the sample)

Согласно представлениям А. Гриффитса, критическое напряжение раскрытия трещины для плоской деформации имеет вид [26]:

$$
\sigma=\sqrt{\frac{2 E \gamma}{\pi(1-\mu) l}},
$$

где $E$ - модуль нормальной упругости; $l$-длина трещины; $\mu$ - коэффициент Пуассона; $\gamma$ - плотность энергии свободной поверхности твердого тела $\left(\gamma=0,1 E b ; b=2,48 \cdot 10^{-8} \mathrm{~cm}-\right.$ межатомное расстояние).

Исходя из этого, длину трещины можно оценивать на основе рис. 6, 7. Так, при нагрузке $P=280 \mathrm{MПа} \mathrm{(рис.} \mathrm{6),} \mathrm{когда} \mathrm{происходит} \mathrm{глубокая}$ (до нуля) релаксация напряжений, длина трещины составляет:

$$
\begin{aligned}
l=\frac{2 E \gamma}{\pi(1-\mu) \sigma^{2}} & =\frac{2\left(205000 \cdot 0,1 \cdot 205000 \cdot 2,48 \cdot 10^{-8}\right)}{3,14(1-0,27) 280^{2}}= \\
& =1,16 \cdot 10^{-3} \mathrm{~cm} \approx 12 \mathrm{м \kappa м} .
\end{aligned}
$$




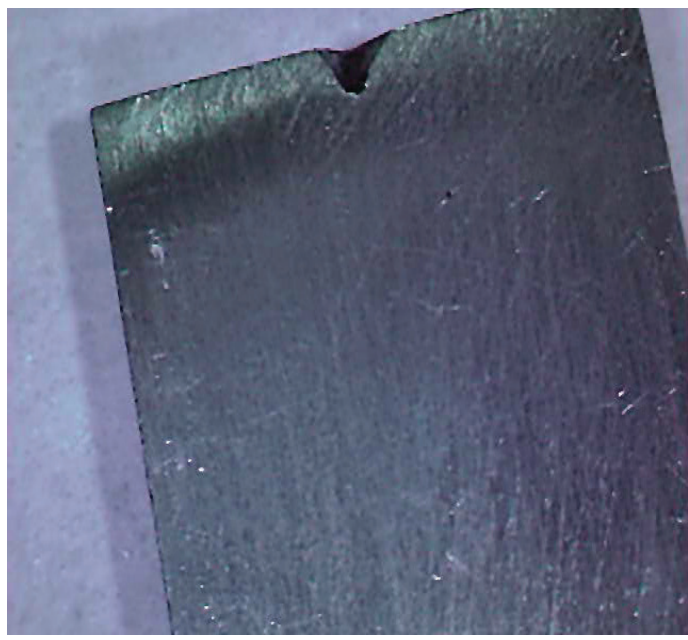

$a$

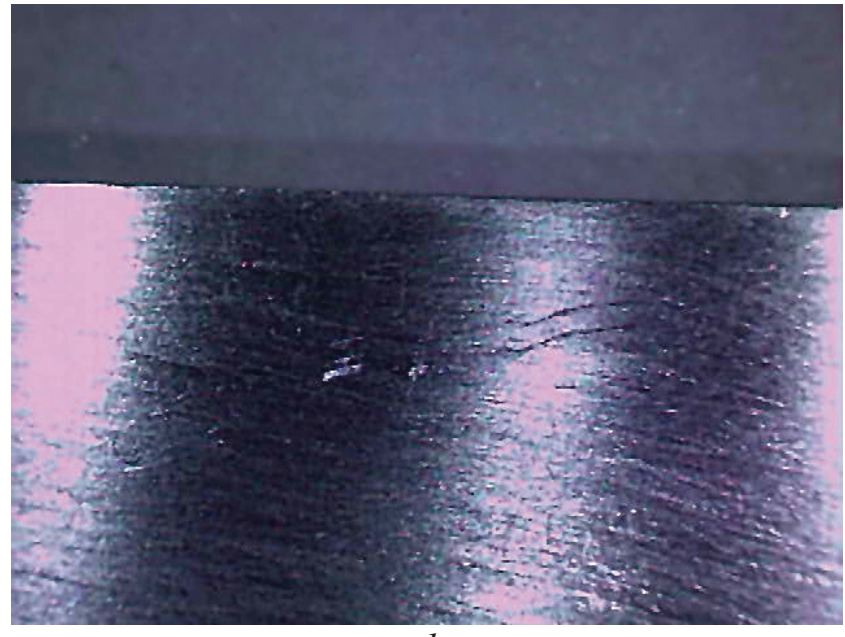

$b$

Рис. 8. Вид деформируемых поверхностей ( $P=75$ МПа); ×10: a) внешняя поверхность; b) внутренняя поверхность

Fig. 8. Type of deformable surfaces $(P=75 \mathrm{MPa}) ; \times 10$ : a) outer surface; $b)$ inner surface

При этом на внутренней поверхности стенки трубы релаксация напряжений наблюдается значительно раньше (75 МПа), чем на наружной, и длина трещины составляет (рис. 7):

$$
\begin{gathered}
l=\frac{2 E \gamma}{\pi(1-\mu) \sigma^{2}}=\frac{2\left(205000 \cdot 0,1 \cdot 205000 \cdot 2,48 \cdot 10^{-8}\right)}{3,14(1-0,27) 75^{2}}= \\
=16,16 \cdot 10^{-3} \mathrm{~cm} \approx 162 \mathrm{M \kappa M.}
\end{gathered}
$$

В целом этот результат соответствует наблюдаемому характеру разрушения: так, на внешней поверхности образца при давлении 75 МПа видимые трещины отсутствуют (рис. $8, a$ ), а на внутренней поверхности видны следы двух наиболее опасных поверхностных протяженных трещин (рис. $8, b$ ).

В процессе циклического деформирования внешняя поверхность образца обнаруживает некоторые отличительные особенности по сравнению с внутренней поверхностью (рис. $9, a, b)$. На внешней поверхности при нагрузке порядка 280 МПа форми-

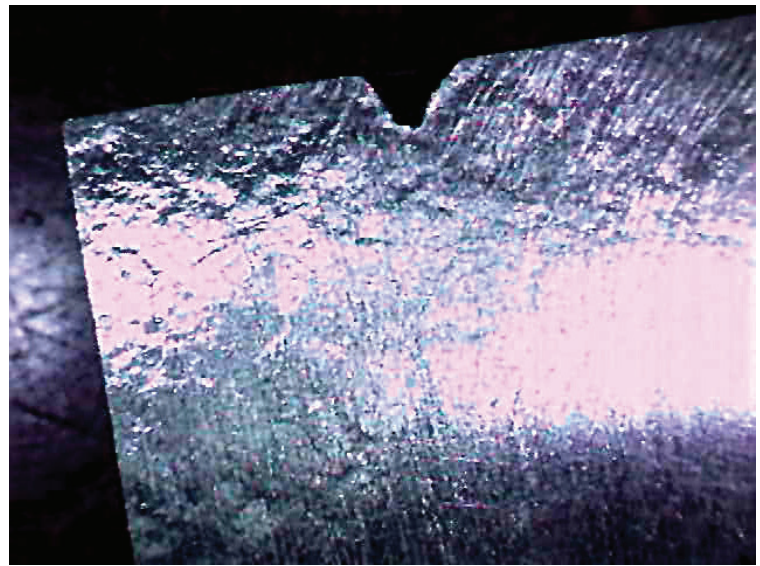

руется ярко выраженная ямочная ячеистая структура, обусловленная процессами экструзии и интрузии (образование впадины). Последние являются местами зарождения трещин. Возникает развитая, распространенная по всей поверхности сетка трещин. Разрушения, наблюдаемые при $280 \mathrm{MПа} \mathrm{(рис.}$ 9), находятся в соответствии с характером релаксаций внутренних напряжений на рис. 6 .

Внутренняя поверхность образца обладает большей жесткостью по отношению к сдвигу (рис. 9, b), она представляется более хрупкой, разрушается при меньших напряжениях (75 МПа) и упрочняется слабо (примерно до 180 МПа, рис. 7). Наружная поверхность более пластична, разрушается при б?льших напряжениях (280 МПа) и упрочняется до 250-350 МПа (рис. 6). Глубина релаксации внутренних напряжений при деформации обеих поверхностей примерно одинакова и составляет порядка 50 МПа.

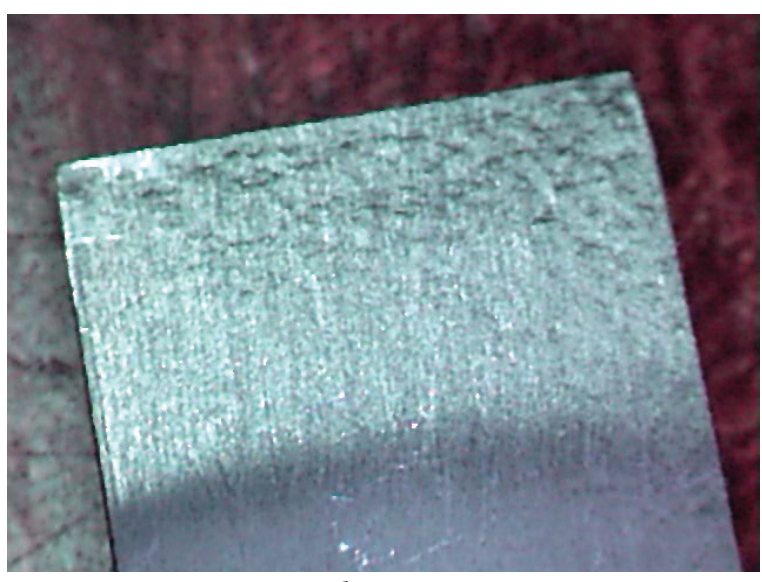

$b$

Рис. 9. Вид деформируемых поверхностей ( $=280$ МПа); $\times 10:$ a) внешняя поверхность; b) внутренняя поверхность

Fig. 9. Type of deformable surfaces $(P=280 \mathrm{MPa}) ; \times 10$ : a) outer surface; $b)$ inner surface 


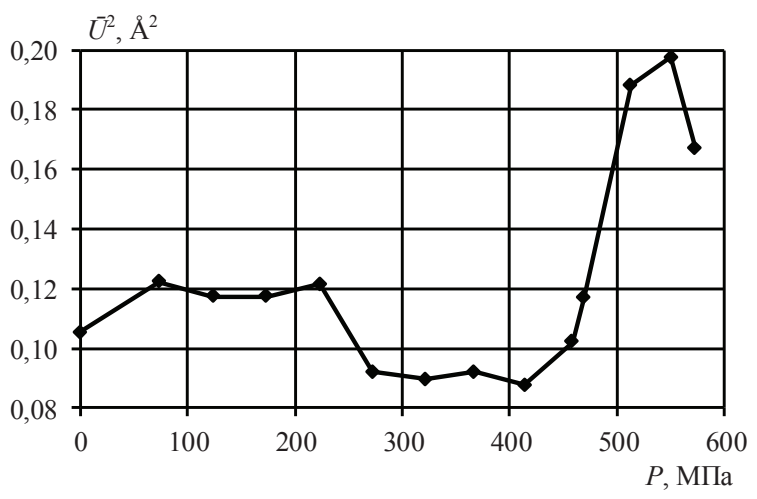

$a$

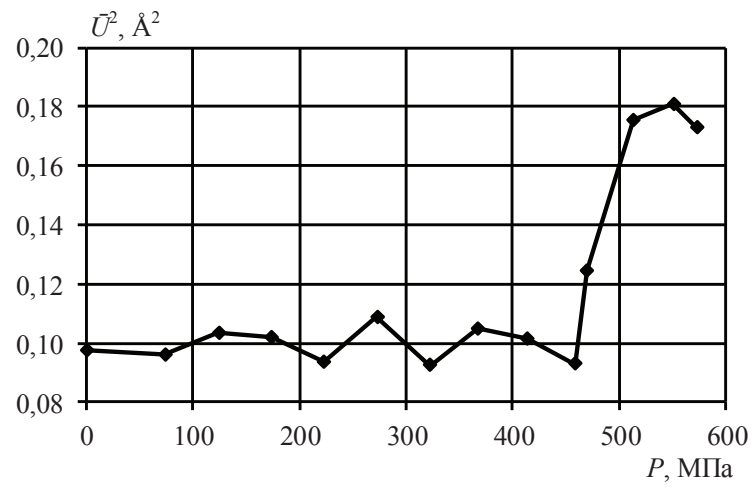

$b$

Рис. 10. Среднеквадратичные отклонения атомов $\left(\bar{U}^{2}\right)$ в зависимости от внешнего давления: a) наружная сторона; b) внутренняя сторона

Fig. 10. Mean square displacement of $\bar{U}^{2}$ atoms as a function of external pressure: a) outer side; $b$ ) inner side

Таким образом, структурным признаком хрупких разрушений является такое смещение $\Delta a / a$, при котором происходит глубокая (до нуля) релаксация напряжений (рис. 6, 7) [27-29].

На рис. 10 представлены зависимости среднеквадратичных смещений атомов при циклическом деформировании.

Структурный признак пластичности - величина среднеквадратичных смещений атомов (рис. $10, a$ ). На более пластичной наружной поверхности до разрушения (до $280 \mathrm{MПа)} \mathrm{среднеквадратичные} \mathrm{смеще-}$ ния $\bar{U}^{2}$ составляют порядка $0,12 \AA^{2}$. При разрыве некоторых межатомных связей, что проявляется релаксацией внутренних напряжений, средняя растянутость межатомных связей уменьшается до $0,09 \AA^{2}$ (рис. $\left.10, a\right)$. При этом увеличиваются силы межатомного взаимодействия (рис. 11, a). При внешнем давлении в 450 МПа амплитуда колебаний атомов возрастает. Амплитуды могут быть настолько значительными, что силы межатомного взаимодействия уже не в состоянии будут удерживать атомы. Атомные связи разрушаются. Растет пористость (например, рис. 5).

Таким образом, на рис. $10, a$ выделяется три участка: первый участок до $280 \mathrm{MПа} \mathrm{соответству-}$ ет пластичному материалу с повышенным ресурсом вытяжки межатомных связей $0,12 \AA^{2}$; второй участок до $450 \mathrm{MПа} \mathrm{соответствует} \mathrm{состоянию}$ хрупких разрушений с частичным разрывом межатомных связей; третий участок от $450 \mathrm{MПа} \mathrm{и}$ выше характеризуется увеличением амплитуды колебаний атомов и соответствует нарастанию пористости.

На менее пластичной внутренней поверхности смещения атомов $\bar{U}^{2}$ колеблются около значения $0,1 \AA^{2}$ (рис. $\left.10, b\right)$. На этой поверхности затруднено пластическое течение, ограничена среднеквадратичная амплитуда атомных смещений и увеличена частота осцилляций атомов $v_{m}: \Theta=\frac{h v_{m}}{k}$. Величина среднеквадратичных отклонений атомов не изменяется в широком диапазоне внешних давлений
вПлоть до 450 МПа и мало отличается от значений $\bar{U}^{2}$ второго участка кривой на рис. $10, a$.

После давлений 450 МПа колебательные амплитуды атомов $\bar{U}^{2}$ повышаются на обеих поверхностях (рис. $10, a, b$ ) и напоминают уже колебания изолированных атомов, амплитуды которых не ограничиваются коллективными связями. Это можно объяснить нарастающей пористостью (рис. 4,5$)$. Возникающие при деформировании дефекты кристаллического строения изменяют спектр частот колебаний атомов за счет нарушения силового взаимодействия атомов при образовании дефекта.

Структурный признак увеличения пористости при ползучести - аномальное увеличение $\bar{U}^{2}$ и уменьшение характеристической температуры (рис. $11, a, b$ ).

Представляет интерес сопоставление зависимостей областей когерентного рассеяния Д от $\bar{U}^{2}$ для образца из околошовной зоны сварного узла (рис. $12, a)$ и образцов из той же стали, изготовленных из прямого участка трубы (рис. $12, b)$. Значения характеристических температур для них приведены в таблице.

таблица. Среднеквадратичные смещения $\bar{U}^{2}$ и характеристические температуры $\Theta$ образцов основного металла из прямых участков трубы (сталь 12X1MФ)

Table. $\quad$ Mean square displacement of $\bar{U}^{2}$ atoms and characteristic temperatures $\Theta$ of base metal specimens from straight pipe sections (steel 12Cr1MoV)

\begin{tabular}{|c|c|c|c|c|}
\hline \multirow{2}{*}{$\begin{array}{c}\text { Исследуемый } \\
\text { образец }\end{array}$} & \multicolumn{2}{|c|}{$\begin{array}{c}\text { Наружная поверхность } \\
\text { Еxternal surface }\end{array}$} & \multicolumn{2}{|c|}{$\begin{array}{c}\text { Ннутренняя поверхность } \\
\text { Internal surface }\end{array}$} \\
\cline { 2 - 5 } Test sample & $\bar{U}^{2}, \AA^{2}$ & $\begin{array}{c}\Theta, \text { град K } \\
\text { (degree kelvin) }\end{array}$ & $\bar{U}^{2}, \AA^{2}$ & $\begin{array}{c}\Theta, \text { град K } \\
\text { (degree kelvin) }\end{array}$ \\
\hline 1 & 0,13357 & 476,7 & 0,09027 & 579,8 \\
\hline $1^{\prime}$ & 0,11370 & 516,6 & 0,12785 & 487,2 \\
\hline 3 & 0,09748 & 558,0 & 0,10879 & 528,2 \\
\hline $3^{\prime}$ & 0,08273 & 605,7 & 0,07120 & 652,9 \\
\hline
\end{tabular}

Образец, изготовленный из зоны сварного шва, обладает повышенными значениями флуктуаций $\bar{U}^{2}$ (рис. $\left.12, a\right)$. 


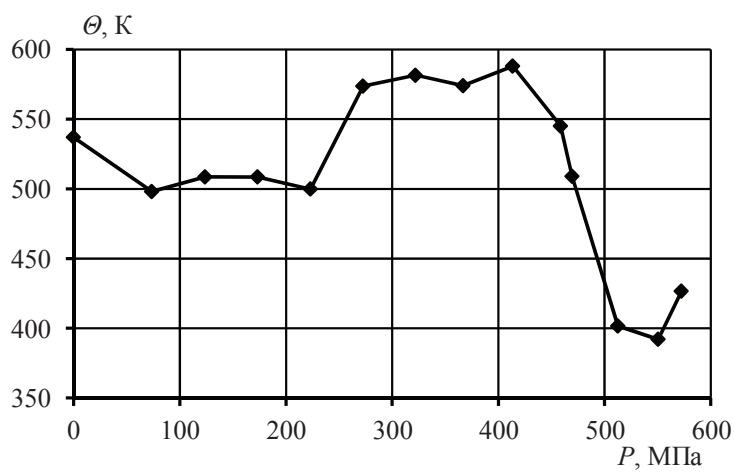

$a$

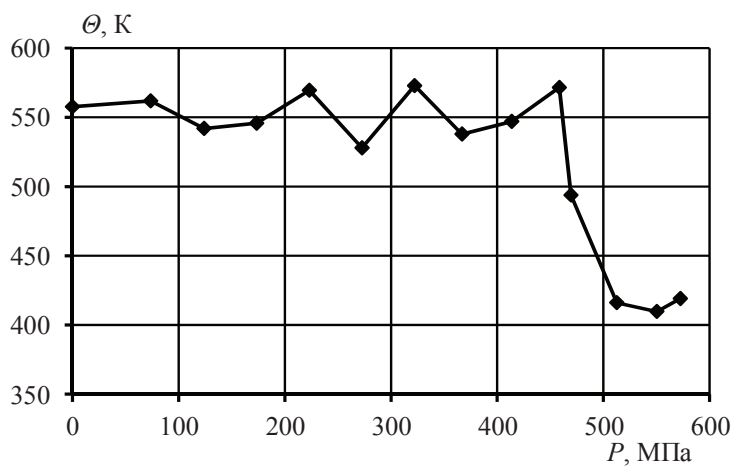

$b$

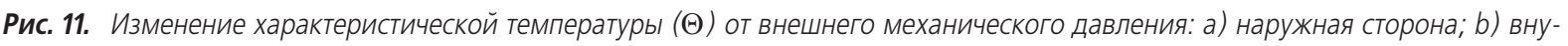
тренняя сторона

Fig. 11. Change of characteristic temperature ( $\Theta)$ from external mechanical pressure: a) outer side; b) inner side

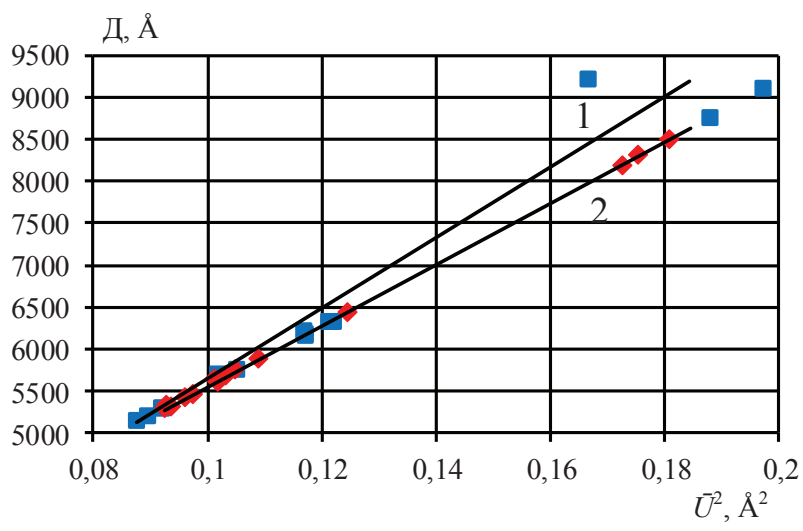

$a$

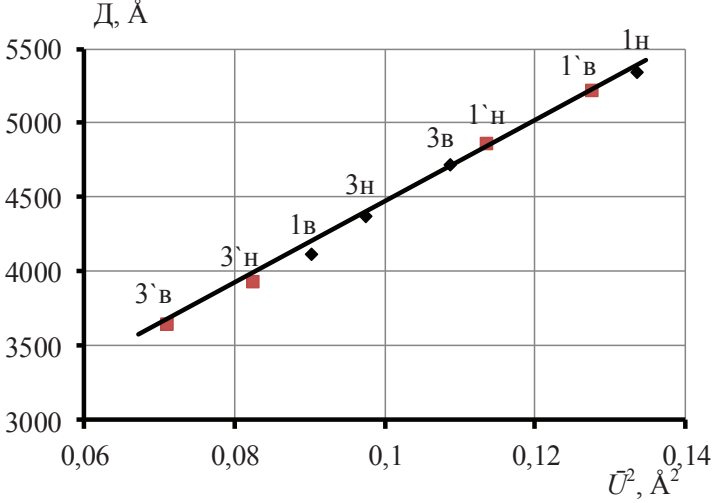

$b$

Рис. 12. Зависимость среднеквадратичного смещения атомов от размера ОКР: а) исследуемый образец из околошовной зоны сварного узла: 1 - наружная поверхность; 2 - внутренняя поверхность; b) основной металл прямого участка трубы: со штрихом - полированные; без штриха - не полированные: н - наружная поверхность; в - внутренняя поверхность

Fig. 12. Mean square displacement of $\bar{U}^{2}$ atoms as a function of the coherent-scattering region size: a) specimen from the weld-affected zone of the weldment: 1 - outer side; 2 - inner side; b) base metal from straight pipe sections: with a stroke - polished; without a stroke-not polished; $\boldsymbol{H}$ - outer side; $\boldsymbol{B}$ - inner side

Если отождествлять накопление поврежденности (пористости) с ростом $\bar{U}^{2}$ (рис. 12 ), то это сравнение позволяет предположить, что околошовная зона сварного узла является изначально поврежденной в результате термического влияния сварочного процесса и фазовой перекристаллизации.

Можно отметить, что среднеквадратичные смещения атомов могут выступать как диагностический признак накопления поврежденности и наступления предельного состояния.

\section{Выводы}

1. Предложена новая методология оценки предельных состояний околошовной зоны сварных соединений на основе использования установленных связей между микроструктурными состояниями, внутренними напряжениями и накоплением структурной повреждаемости.
2. Колебательная амплитуда атомов $\bar{U}^{2}$ может служить диагностическим признаком наступления предельного состояния.

3. Признаком хрупкого разрушения является глубокая (до нуля) релаксация внутренних напряжений I рода.

4. Структурный признак пластичности проявляется повышенной амплитудой атомных смещений $\bar{U}^{2}$.

5. Структурным признаком пористости является аномальное повышение колебательной амплитуды атомов $\bar{U}^{2}$ и уменьшение характеристической температуры.

Работа выполнена при поддержке РФФИ «Фундаментальные основы инженерных наук (проект № 15-08-99544а в 20142., № 18-08-01265 в 20018 г.) 


\section{СПИСОК ЛИТЕРАТУРЫ}

1. Reasons for and nature of weld joints failure in hot reheat steam lines of 800-MW power units / A.E. Anokhov, E.A. Grin, T.V. Perevezentseva, I.V. Fedina // Thermal Engineering. - 2009. V. 56. - № 2. - P. 113-119.

2. Хромченко Ф.А. Сварные соединения трубопроводов. Диагностика и ресурс. В 2-х т. Т. 1. - М.: Теплотехник, 2010. - 304 с.

3. Failure analysis of a steam pipe weld used in power generation plant / Chu Qiaoling, Zhang Min, Li Jihong, Chen Yinni, Luo Hailong, Wang Qiang // Engineering Failure Analysis. - 2014. V. 44. - P. 363-370.

4. Determination of mechanical and fracture properties of laser beam welded steel joints / G. Cam, S. Erim, Ç. Yeni, M. Koçak // Welding Journal. - 1999. - V. 78. - № 6. - P. 193-201.

5. Measures for Ensuring Reliable Operation of the Welded Joint Connecting the Reactor Coolant Circuit's Header to the Shell of a Steam Generator Used at a VVER-1000 Reactor-Based Nuclear Power Station / S.A. Kharchenko, N.B. Trunov, N.F., Korotaev S.L. Lyakishev // Thermal Engineering. - 2011. - V. 58. № 3. - P. 208-214.

6. Huang Tengfei, Zhang Guihe, Liu Feihua. Design, manufacturing and repair of tube-to-tube sheet welds of steam generators of CPR1000 units // Nuclear Engineering and Design. - 2018. V. $333 .-$ P. $55-62$.

7. Sorrentino S. Welding technologies for ultra-supercritical power plant materials // Materials for Ultra-Supercritical and Advanced Ultra-Supercritical Power Plants. - 2017. - P. 247-319. URL: http://www.sciencedirect.com/science/book/ 9780081005521 (дата обращения: 03.10.2018).

8. РД 10-249-98. Нормы расчета на прочность стационарных котлов и трубопроводов пара и горячей воды. - М.: ЗАО НТЦ ПБ, 2010. -270 c.

9. Николаев Г.А. Расчет сварных соединений и прочность сварных конструкций. - М.: Высшая школа, 1965. - 451 с.

10. Zaefferer S., Ohlert J., Bleck W. A study of microstructure, transformation mechanisms and correlation between microstructure and mechanical properties of a low alloyed TRIP steel // Acta Materialia. - 2004. - V. 52. - № 9. - P. 2765-2778.

11. Minin S.I., Trofimov A.I., Trofimov M.A. The technology of thermal welding of the circulation piping of NPPS containing the influence of ultrasound // Nuclear Energy and Technology. 2017. - V. 3. - № 1. - P. 6-9.

12. Welding residual stresses as needed for the prediction of fatigue crack propagation and fatigue strength / J. Hensel, T. NitschkePagel, D. Tchoffo Ngoula, H.-Th. Beier, D. Tchuindjang, U. Zerbst // Engineering Fracture Mechanics. - 2018. V. 198. - P. 123-141.

13. Popov A.B. Main factors causing damage to the high-temperature heating surfaces used in power-generating boilers // Thermal Engineering. - 2011. - V. 58. - № 2. - P. 101-108.
14. Capello E. Residual stresses in turning: P. I: Influence of process parameters // Journal of Materials Processing Technology. 2005. - V. 160. - № 2. - P. 221-228.

15. Горелик С.С., Скаков Ю.А., Расторгуев Л.Н. Ренттенографический и электронно-оптический анализ. - М.: МИСИС, 2002. - 360 с.

16. Русаков А.А. Рентгенография металлов. - М.: Атомиздат, 1977. $-480 \mathrm{c}$.

17. Friedel J., Gullity B.D., Crussard C. Study of the surface tension of a grain boundary in a metal as a function of the orientation of the two grains which the boundary separates // Acta Metallurgica. - 1953. - V. 1. - P. 79-92.

18. Pan Jian-hua, Fan Zhi-cao, Zong Ning-sheng. Research on weld cracking of TP321H stainless steel pipeline under elevated temperature // International Journal of Pressure Vessels and Piping. 2016. - V. 148. - P. 1-8.

19. Регель В.Р., Слуцкер А.И., Тамашевский Э.Е. Кинетическая природа прочности твердых тел. - М.: Наука, 1974. - 560 с.

20. Dieter G.E. Mechanical Metallurgy. - New York: McGraw-Hill Book Company, 1961. $-615 \mathrm{p}$.

21. Atienza J.M., Elices M. Influence of residual stresses in the stress relaxation of cold drawn wires // Materials and Structures. 2004. - V. 37. - № 5. - P. 301-304.

22. Residual stress driven creep cracking in AISI Type 316 stainless steel / M. Turski, P.J. Bouchard, A. Steuwer, P.J. Withers // Acta Materialia. - 2008. - V. 56. - № 14. - P. 3598-3612.

23. Feltham P. Creep and stress relaxation in alpha-brass at low temperatures // Philosophical Magazine. - 1961. - V. 6 (62). - P. 259-270.

24. A study of the generation and creep relaxation of triaxial residual stresses in stainless steel / S. Hossain, C.E. Truman, D.J. Smith, R.L. Peng, U. Stuhr // International Journal of Solids and Structures. - 2007. - V. 44. - № 9. - P. 3004-3020.

25. Structural Instability in the Early Decomposition of Supersaturated Austenite Solid Solution / A.A. Makeev, L.L. Lyubimova, A.S. Zavorin, A.A. Tashlykov // Steel in Translation. - 2009. V. 39. - № 12. - P. 1048-1055.

26. Griffith A.A. The phenomena of rupture and flow in solids // Philosophical Transactions of the Royal Society of London. - 1920. Series A, 221. - P. 163-168.

27. Способ установления состояния предразрушения конструкционного изделия: пат. Рос. Федерация № 2613486; заявл. 21.12.15; опубл. 16.03 .17 , Бюл. № 8. - 18 c.

28. The effect of thermo-mechanical loading on fracture-related parameters of austenitic steel / L.L. Lyubimova, A.A. Tashlykov, R.B. Tabakaev, A.A. Levin, A.Y. Popov // Engineering Failure Analysis. - 2017. - V. 81. - P. 45-56.

29. Changes in the State of Heat-Resistant Steel Induced by Repeated Hot Deformation / L.L. Lyubimova, R.N. Fisenko, A.A. Tashlykov, R.B. Tabakaev // Metals and Materials International. 2018. - V. 24. - № 1. - P. 121-129.

Поступила 06.10.2018 г.

\section{Информация об авторах}

Заворин A.C., доктор технических наук, профессор научно-образовательного центра И.Н. Бутакова Инженерной школы энергетики Национального исследовательского Томского политехнического университета.

Любимова Л.Л., кандидат технических наук, доцент научно-образовательного центра И.Н. Бутакова Инженерной школы энергетики Национального исследовательского Томского политехнического университета.

Tашлыков A.A., кандидат технических наук, доцент научно-образовательного центра И.Н. Бутакова Инженерной школы энергетики Национального исследовательского Томского политехнического университета.

Буваков $\boldsymbol{K} . \boldsymbol{B .}$., кандидат технических наук, доцент научно-образовательного центра И.Н. Бутакова Инженерной школы энергетики Национального исследовательского Томского политехнического университета. 
UDC 621.181:621.791:620.1

\title{
STRUCTURAL ASPECTS OF CYCLICAL STRENGTH OF A SUPERHEATER WELDMENT
}

\author{
Alexander S. Zavorin', \\ zavorin@tpu.ru
}

\section{Lyudmila L. Lyubimova', ||@tpu.ru}

\author{
Alexander A. Tashlykov', \\ tashlykov@tpu.ru \\ Konstantin V. Buvakov', \\ kvb@tpu.ru \\ 1 National Research Tomsk Polytechnic University, \\ 30, Lenin Avenue, Tomsk, 634050, Russia.
}

The relevance of researching power generator weldments is associated with the necessity of increasing the operational reliability of the heat-power equipment frequently damaged and most critical units. One of the working efficiency increasing factors is the consideration of internal structural stresses, formation mechanism of which is associated with the structural heterogeneity developing in exploitation and resource structural degradation leading to destruction.

The aim of the research is to establish interrelationships between the structural damage accumulation, internal stresses and cracking under the mechanical cyclic loads influence.

Subject: welded superheater unit made of low-alloy heat-resistant steel 12CriMoV.

Methods: physical modeling of operating conditions by external mechanical cyclic deformation, microscopic surface morphology research, $x$-ray diffraction of deformed samples, evaluation of internal structural stresses, mean square atoms displacement during deformation and characteristic temperature.

Results. The paper demonstrates the role and the influence of the cyclic load on fracture. It means that wave periodic deformation "hardening-softening" and the cyclic variation of internal structural stresses are the most important. It was established that under variable cyclic load conditions the periodic internal stresses relaxation is typical. This process corresponds to the rupture of interatomic bonds and formation of a microstructural short crack. According to relaxation conditions, based on Griffith ideas, the authors have determined the critical crack opening stress. The values of mean square displacement of atoms and the characteristic temperature during cyclic deformation reflect the structural features of plasticity, porosity and brittle state.

Conclusions. The paper introduces a new methodology for estimating critical states of the weld-affected zone based on the use of relations between internal stresses and the structural damage accumulation. It is shown that internal stresses, vibrational amplitude of $\bar{U}^{2}$ atoms and characteristic temperature can be a sign of structural transformation diagnostic: the brittle fracture sign is a deep (to zero) relaxation of the first kind internal stresses; the structural plasticity sign is an increased amplitude of atomic $\bar{U}^{2}$ displacements; and the structural porosity feature is an abnormal increase in the vibrational atoms amplitude and characteristic temperature decrease.

\section{Key words:}

Weldment, steel, superheater, internal stresses, mechanical cyclic loads, structural degradation, crack formation, cyclical strength, atomic mean square displacement, characteristic temperature.

This work was supported by the Russian Foundation for Basic Research «Fundamentals of Engineering Sciences» (project No.15-08-99544a in 2014, No.18-08-01265 in 2018).

\section{REFERENCES}

1. Anokhov A.E., Grin E.A., Perevezentseva T.V., Fedina I.V. Reasons for and nature of weld joints failure in hot reheat steam lines of 800-MW power units. Thermal Engineering, 2009, vol. 56, no. 2, pp. 113-119. In Rus.

2. Khromchenko F.A. Svarnye soedineniya truboprovodov. Diagnostika i resurs [Welded connections of pipelines. Diagnostics and resource]. Moscow, Teplotekhnik Publ., 2010. Vol. 1, 304 p.

3. Chu Qiaoling, Zhang Min, Li Jihong, Chen Yinni, Luo Hailong, Wang Qiang. Failure analysis of a steam pipe weld used in power generation plant. Engineering Failure Analysis, 2014, vol. 44, pp. 363-370.

4. Cam G., Erim S., Yeni Ç., Koçak M. Determination of mechanical and fracture properties of laser beam welded steel joints. Welding Journal, 1999, vol. 78, no. 6, pp. 193-201.

5. Kharchenko S.A., Trunov N.B., Korotaev N.F., Lyakishev S.L. Measures for Ensuring Reliable Operation of the Welded Joint Connecting the Reactor Coolant Circuit's Header to the Shell of a
Steam Generator Used at a VVER-1000 Reactor-Based Nuclear Power Station. Thermal Engineering, 2011, vol. 58, no. 3, pp. 208-214. In Rus.

6. Huang T., Zhang G., Liu F. Design, manufacturing and repair of tube-to-tubesheet welds of steam generators of CPR1000 units. Nuclear Engineering and Design, 2018, vol. 333, pp. 55-62.

7. Sorrentino S. Welding technologies for ultra-supercritical power plant materials. Materials for Ultra-Supercritical and Advanced Ultra-Supercritical Power Plants, 2017, pp. 247-319. Available at: http://www.sciencedirect.com/science/book/9780081005521 (accessed 3 0ctober 2018).

8. RD 10-249-98. Normy rascheta na prochnost statsionarnykh kotlov i truboprovodov para i goryachey vody [Standards for strength calculation of stationary boilers and steam and hot water pipelines]. Moscow, ZA0 NTC PB Publ., 2010. 270 p.

9. Nikolaev G.A. Raschet svarnykh soedineniy i prochnost svarnykh konstruktsy [Calculation of welded joints and strength of welded structures]. Moscow, Vysshaya shkola Publ., 1965. 451 p. 
10. Zaefferer S., Ohlert J., Bleck W. A study of microstructure, transformation mechanisms and correlation between microstructure and mechanical properties of a low alloyed TRIP steel. Acta Materialia, 2004, vol. 52, no. 9, pp. 2765-2778.

11. Minin S.I., Trofimov A.I., Trofimov M.A. The technology of thermal welding of the circulation piping of NPPS containing the influence of ultrasound. Nuclear Energy and Technology, 2017, vol. 3, no. 1, pp. 6-9.

12. Hensel J., Nitschke-Pagel T., Tchoffo Ngoula D., Beier H.-Th., Tchuindjang D., Zerbst U. Welding residual stresses as needed for the prediction of fatigue crack propagation and fatigue strength. Engineering Fracture Mechanics, 2018, vol. 198, pp. 123-141.

13. Popov A.B. Main factors causing damage to the high-temperature heating surfaces used in power-generating boilers. Thermal Engineering, 2011, vol. 58, no. 2, pp. 101-108. In Rus.

14. Capello E. Residual stresses in turning: P. I: Influence of process parameters. Journal of Materials Processing Technology, 2005, no. 2, pp. 221-228.

15. Gorelik S.S., Skakov Yu.A., Rastorguev L.N. Rentgenografichesky i elektronno-optichesky analiz [X-ray and electron-optical analysis]. Moscow, MISIS Publ., 2002. 360 p.

16. Rusakov A.A. Rentgenografiya metallov [X-ray diffraction of metals]. Moscow, Atomizdat Publ., 1977. 480 p.

17. Friedel J., Gullity B. D., Crussard C. Study of the surface tension of a grain boundary in a metal as a function of the orientation of the two grains which the boundary separates. Acta Materialia, 1953, vol. 1, pp. 79-92.

18. Pan Jian-hua, Fan Zhi-cao, Zong Ning-sheng. Research on weld cracking of TP321H stainless steel pipeline under elevated temperature. International Journal of Pressure Vessels and Piping, 2016, vol. 148, pp. 1-8.

19. Regel V.R., Slucker A.I., Tamashevsky E.E. Kineticheskaya priroda prochnosti tverdykh tel [Kinetic nature of solid strength]. Moscow, Nauka Publ., 1974. 560 p.

20. Dieter G.E. Mechanical Metallurgy. New York, McGraw-Hill Book Company Publ., 1961. 615 p.
21. Atienza J.M., Elices M. Influence of residual stresses in the stress relaxation of cold drawn wires. Materials and Structures, 2004, vol. 37, no. 5, pp. 301-304.

22. Turski M., Bouchard P.J., Steuwer A., Withers P.J. Residual stress driven creep cracking in AISI Type 316 stainless steel. Acta Materialia, 2008, vol. 56, no. 14, pp. 3598-3612.

23. Feltham P. Creep and stress relaxation in alpha-brass at low temperatures. Philosophical Magazine, 1961, vol. 6 (62), pp. 259-270.

24. Hossain S., Truman C.E., Smith D.J., Peng R.L., Stuhr U. A study of the generation and creep relaxation of triaxial residual stresses in stainless steel. International Journal of Solids and Structures, 2007, vol. 44, no. 9, pp. 3004-3020.

25. Makeev A.A., Lyubimova L.L., Zavorin A.S., Tashlykov A.A. Structural Instability in the Early Decomposition of Supersaturated Austenite Solid Solution. Steel in Translation, 2009, vol. 39, no. 12, pp. 1048-1055.

26. Griffith A.A. The phenomena of rupture and flow in solids. Philosophical Transactions of the Royal Society of London, 1920, Series A, 221, pp. 163-168.

27. Lyubimova L.L., Zavorin A.S., Tashlykov A.A., Tabakaev R.B. Sposob ustanovleniya sostoyaniya predrazrusheniya konstruktsionnogo izdeliya [The manufacturing method for a state of a structural work-piece pre-destruction]. Patent RF, no. 2613486, 2017.

28. Lyubimova L.L., Tashlykov A.A., Tabakaev R.B., Levin A.A., Popov A.Y. The effect of thermo-mechanical loading on fracturerelated parameters of austenitic steel. Engineering Failure Analysis, 2017, vol. 81, pp. 45-56.

29. Lyubimova L.L., Fisenko R.N., Tashlykov A.A., Tabakaev R.B. Changes in the State of Heat-Resistant Steel Induced by Repeated Hot Deformation. Metals and Materials International, 2018, vol. 24, no. 1, pp. 121-129.

Received: 6 October 2018.

Information about the authors

Alexander S. Zavorin, Dr. Sc., professor, National Research Tomsk Polytechnic University.

Lyudmila L. Lyubimova, Cand. Sc., associate professor, National Research Tomsk Polytechnic University.

Alexander A. Tashlykov, Cand. Sc., associate professor, National Research Tomsk Polytechnic University.

Konstantin V. Buvakov, Cand. Sc., associate professor, National Research Tomsk Polytechnic University. 